\title{
Giant cell angiofibroma misdiagnosed as a vascular malformation and treated with absolute alcohol for one year: a case report and review of the literature
}

Yue He ${ }^{1 *}$, Chenping Zhang ${ }^{1}$, Guanglong Liu', Zhuowei Tian', Lizhen Wang ${ }^{2}$ and Evagelos Kalfarentzos ${ }^{1}$

\begin{abstract}
Purpose: To present the clinical, imaging, pathological and immunohistochemical features of giant cell angiofibroma (GCA).

Case presentation: In this paper we report an atypical case of a GCA extending from the parotid to the parapharyngeal space. The lesion was being treated as a vascular malformation for one year prior to surgical removal. We summarize the clinical manifestations, imaging, pathological and molecular features of this rare disease.

After complete surgical removal of the tumor, immunohistochemical analysis revealed strong positivity for the mesenchymal markers vimentin, CD34, CD31 and CD99 in neoplastic cells. Tumor proliferation antigen marker Ki67 was partly positive ( $<5 \%$ of cells). Tumor cells were negative for muscle-specific actin, epithelial membrane antigen, smooth muscle actin, cytokeratin pan, S100, desmin, glial fibrillary acidic protein, myogenin, MyoD1 and F8. The morphological and immunohistochemical profile was consistent with the diagnosis of GCA.

Conclusion: GCA is a rare soft tissue tumor that can easily be misdiagnosed in the clinical preoperative setting. In view of the clinical, pathological and molecular features of the tumor, complete surgical removal is the current optimal treatment option, providing accurate diagnosis and low to minimal recurrence rate.
\end{abstract}

Keywords: Giant cell angiofibroma, Parotid gland, Parapharyngeal space, Immunohistochemistry

\section{Background}

Giant cell angiofibroma (GCA) was first described by Dei Tos as a rare soft tissue tumor of the orbit [1]. Since the initial report of the disease in orbital tissue, GCA has been reported in a variety of other extraorbital sites [2-8]. GCA is a benign, mesenchymal lesion showing histological features intermediate between, but distinct from, solitary fibrous tumor (SFT) and giant cell fibroblastoma (GCF) of soft tissue [5].

In this paper, we report a rare case of a patient with a large GCA extending from the right parotid gland to the homolateral parapharyngeal space that was being treated

\footnotetext{
* Correspondence: wyuehe@gmail.com

'Department of Oral and Maxillofacial-Head and Neck Oncology, Ninth People's Hospital, Shanghai Jiao Tong University School of Medicine, 639, Zhi Zao Ju Road, Shanghai 200011, People's Republic of China

Full list of author information is available at the end of the article
}

as a vascular malformation for a year prior to surgical removal. The clinical findings, diagnostic procedure followed, treatment and histopathological/immunohistochemical findings are also outlined in this paper.

\section{Case presentation}

Clinical examination

A 29-year-old male was referred to our outpatient department with a 24-month history of a slowly growing, painless mass of the right parotid gland and submandibular region. He also complained of mild discomfort during swallowing. The patient's medical history was unremarkable with no previous history of surgery. At the time, he was diagnosed as having a vascular malformation of the right parotid-parapharyngeal region and he had been under treatment for one year with 
sclerotherapy with no evidence of improvement or stabilization of the tumor.

Extraoral physical examination at the time of referral was suggestive of a large, mobile, smooth-surfaced, fibroelastic soft tissue mass, which was palpated in the lower pole of the right parotid gland. The lesion extended subcutaneously along the homolateral submandibular and pharyngeal spaces (Figure 1A). On palpation the mass was painless with no evidence of any emanating pulsation. Neurological examination of cranial nerves was normal, and no palpable lymph nodes were present in the neck, supraclavicular or axillary regions.

Upon intraoral examination, there was evidence of the mass bulging to the right oral pharynx with subsequent obliteration of the palatoglossal arch. Deviation of the uvula and soft palate was not evident during opening of the mouth. Covering mucosa of the region was normal in color and texture (Figure 1B). The remainder of the head and neck clinical examination was normal.

\section{Diagnostic tests}

The patient was initially clinically diagnosed with a vascular malformation (VM) of the parotid, submandibular and parapharyngeal spaces based on computed tomography $(\mathrm{CT})$ scan, magnetic resonance imaging (MRI) of the head and neck and a fine needle aspiration biopsy that was indicative of intense hemorrhagic necrosis. At the time, the mass measured approximately $58 \times 25 \times$ $47 \mathrm{~mm}$ (Figure 2A, B). After unsuccessful treatment with sclerotherapy for one year, a new MRI was performed revealing slow growth of the tumor. MRI was suggestive of an inhomogeneous mass which measured $81 \times 34 \times$ $67 \mathrm{~mm}$ and extended from the right parotid gland into the right parapharyngeal space. (Figure 2C, D). The signal of the mass was isointense to the circumambient soft tissue in T1W1. Hyperintense signal was demonstrated in T2W1 and T2W1 (Fat suppression) images. There was no sign of infiltration to adjacent soft tissue and no bone erosion was identified on imaging studies. MRI findings were suggestive of a benign parotid gland tumor with involvement of the homolateral submandibular and parapharyngeal spaces.

\section{Treatment}

As mentioned above, clinical history and physical examination were suggestive initially of the diagnosis of VM. Thus, the patient was first treated with a regimen of percutaneous sclerotherapy using ethanol and lauromacrogol for a period of one year without any complications. During this period, no response to sclerotherapy
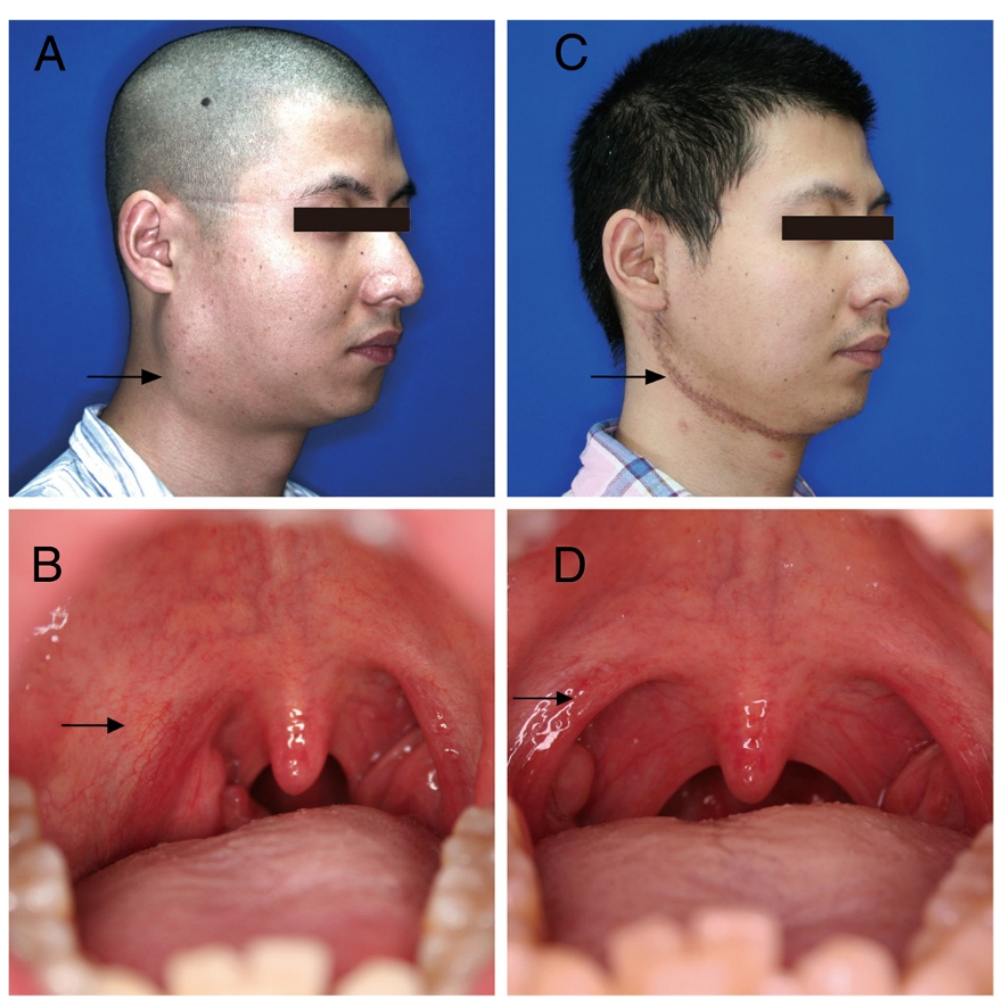

Figure 1 Clinical view of the tumor. (A) Preoperative extraoral clinical view of the patient. (B) Preoperative intraoral view of the patient. (C) Postoperative extraoral clinical view of the patient. (D) Postoperative intraoral clinical view of the patient. 

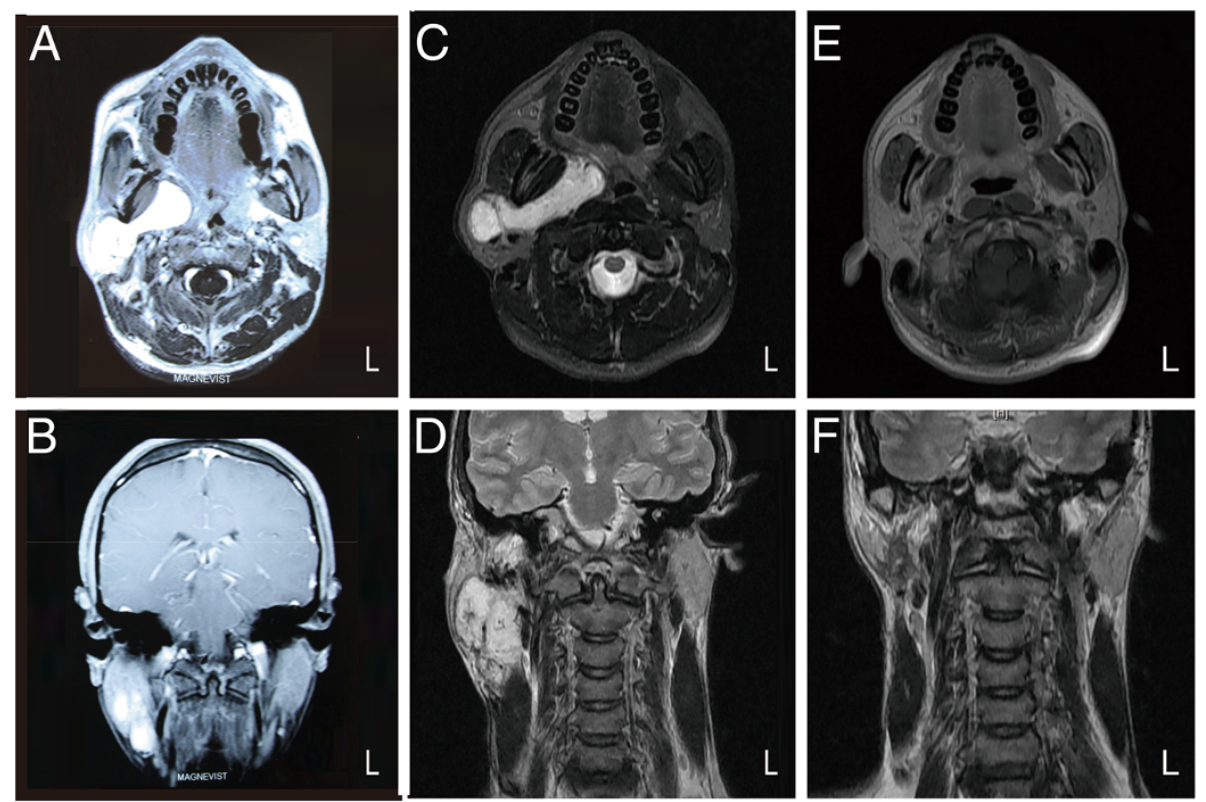

Figure 2 Giant cell angiofibroma. (A) Axial T1-weighted MRI of the tumor prior to sclerotherapy. (B) Coronal T1-weighted MRI of the tumor prior to sclerotherapy. (C) Preoperative axial T2-weighted MRI of the tumor after sclerotherapy. (D) Preoperative coronal T1-weighted MRI of the tumor after sclerotherapy. (E) Postoperative axial T1-weighted MRI at six months after resection showing no signs of recurrence. (F) Postoperative coronal T1-weighted MRI at six months after resection showing no signs of recurrence.

was evident; furthermore the tumor slowly increased in size with no other significant changes in regard to its clinical and radiological consistency.

Since sclerotherapy treatment proved unsuccessful, after discussion and written consent from the patient the decision to surgically remove the tumor was made. The patient was prepared for general anesthesia via nasotracheal intubation. Access to the tumor was feasible through a preauricular incision with a submandibular extension. A skin flap above the parotid fascia and incorporating the platysma muscle was raised with subsequent identification and preservation of the cervicofacial branch of the facial nerve. The tumor appeared to have no obvious infiltration to the surrounding tissues and was excised completely. There was no major intraoperative bleeding. The residual surgical defect was not reconstructed and the wound was closed in layers under suction drainage.

Macroscopically, the tumor was shaped like a dumbbell and contained a single cystic space filled with a yellow-brown sticky fluid.

The patient's postoperative healing was uneventful. He is on a regular follow up program and six months postoperatively there is no sign of recurrence (Figure 1C, D, Figure 2E, F).

\section{Histopathology}

Light microscopy examination of the lesion showed increased cellularity, with spindled and elongated cells intermingled with variable amounts of capillary-sized blood vessels, pseudovascular spaces, and floret-like multinucleated giant cells (Figure 3). At high magnification, the spindle-shaped tumor cells had plump nuclei and pale eosinophilic cytoplasm. No mitoses were identified.

Immunohistochemistry was performed and the mesenchymal markers vimentin, CD31, CD34, CD99 were positive in spindle cells and giant cells. Tumor proliferation antigen marker Ki67 was partly positive $(<5 \%$ of cells). Muscle-specific actin (MSA), epithelial membrane antigen (EMA), smooth muscle actin (SMA), cytokeratin pan (CKP), S100, desmin, CD31, glial fibrillary acidic protein (GFAP), myogenin, MyoD1 and F8 were negative (Figure 4). The morphology and immunohistochemical profile were consistent with the diagnosis of GCA.

\section{Discussion}

GCA is a rare tumor of soft tissues. Although it was firstly described as a distinct orbital neoplasm by Dei Tos [1], until now there are approximately 26 various extraorbital site cases reported in the literature [2-18] (Table 1). By taking into account the orbital GCA cases and extraorbital head and neck cases we could assume that GCA may have a predilection for the head and neck region.

The tumor may grow rapidly, simulating malignancy, or may have an indolent course with slow growth over many years. GCA most often occurs in middle-aged 


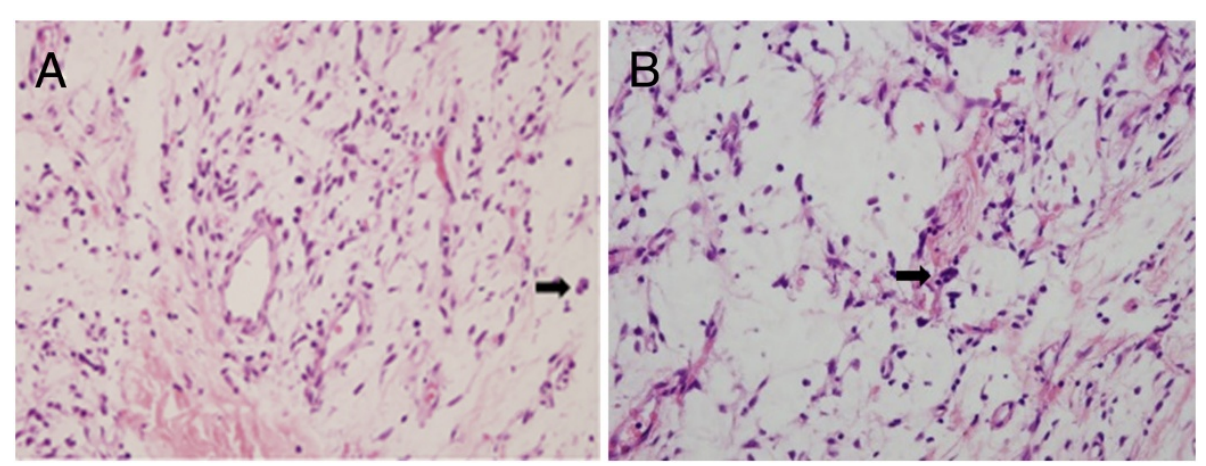

Figure 3 Histopathological examination. (A) and (B) Microscopic examination revealed a circumscribed, richly vascularized, patternless spindle cell proliferation containing pseudovascular spaces and floret-like multinucleated giant cells (arrow), sometimes lining the pseudovascular spaces, in a variably collagenous or myxoid stroma. (H\&Ex400).

adults (median age 45 years). In regard to sex predilection of this tumor, some reports suggest that orbital GCA is more common in men, while extraorbital locations are more common in women [6].

Gonzalez-Perez et al. were the first to report a GCA of the parapharyngeal region in a 25-year-old female patient [6]. Both GCA cases presented with similar clinical characteristics of a benign non-infiltrative slow growing tumor. In both cases a differential diagnosis of parotid gland, fibrous or vascular tumor was made. In our case, the more cystic (pseudocystic) nature of the tumor in addition to the lack of other evidence from the fine needle aspiration biopsy, led to the misdiagnosis of the tumor as a VM. Despite the positive results for CD31 in our case, histological and immunohistochemical findings were also similar in both cases.
The pathogenesis of the tumor remains unclear. There are currently two papers in the literature trying to associate an orbital case of GCA with mutations on chromosome 6q13 [18] and another extraorbital case with an associated translocation $\mathrm{t}(12 ; 17)$ [14].

In the clinical setting during preoperative management, diagnosis of GCA may be virtually impossible. Besides the fact that GCA is an extremely rare tumor it also shares many clinical and radiological features with other soft tissue tumors of the head and neck. Furthermore the pseudovascular spaces that are present in large GCAs may associate it with tumors of vascular origin, as in our case. The multitude of soft tissue tumors of the region that need to be differentially diagnosed from GCA, can be roughly depicted in an abbreviated summary of the World Health Organization (WHO) system

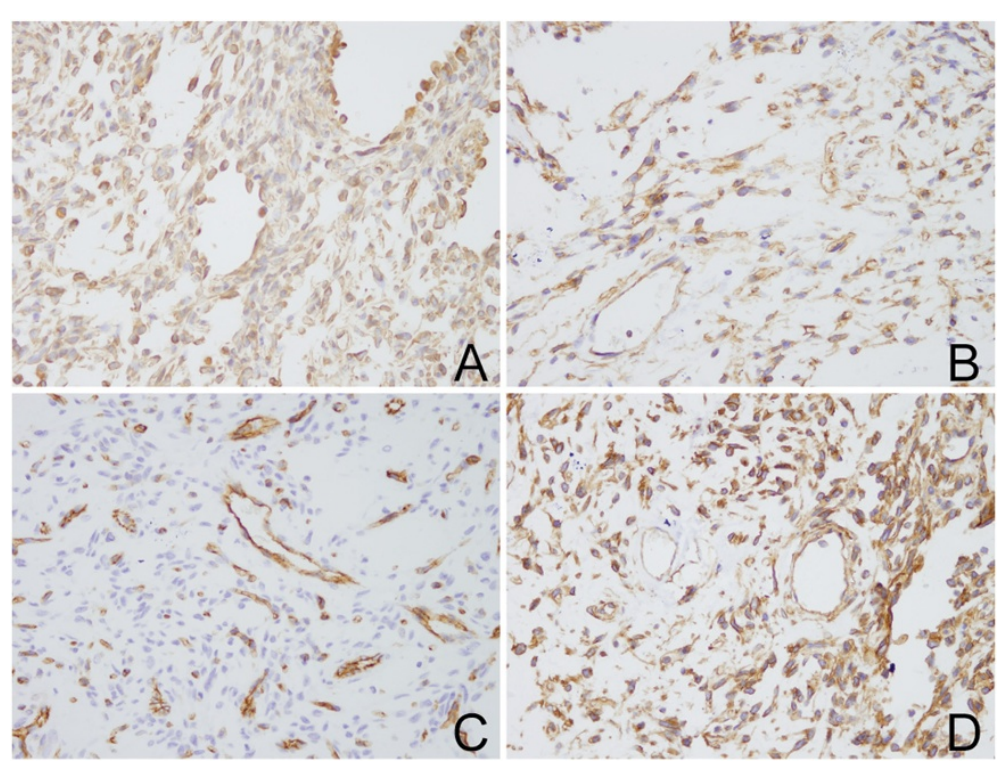

Figure 4 Immunohistochemical analysis revealed strong positivity for the mesenchymal markers. (A) Vimentin. (B) CD34. (C) CD31. (D) CD99. 


\begin{tabular}{|c|c|c|c|}
\hline Head and neck area & & Other anatomic areas & \\
\hline Subcutaneous neck area & 1 & Cutaneous (thigh) & 1 \\
\hline Occipital region & 2 & Hip & 1 \\
\hline Retroauricular area & 1 & Forearm & 1 \\
\hline Parotid & 1 & Vulva & 1 \\
\hline Submandibular region & 1 & Retroperitoneum & 1 \\
\hline Parapharyngeal space & 1 & Back & 3 \\
\hline Oral buccal mucosa & 3 & Mediastinum & 1 \\
\hline Tongue & 1 & Axilla & 1 \\
\hline Vocal cord & 2 & Groin/Inguinal region & 3 \\
\hline
\end{tabular}

Source: References [2-18].

for classifying soft tissue tumors on the basis of tissue type and biologic potential (Table 2) [19].

Vascular malformation is a collective term for many different disorders of the vasculature. It can be a disorder of capillaries, arteries, veins and lymphatics or a combination of those. A VM consists of a clew of deformed vessels, due to an error in vascular development. However, endothelial turnover is stable in these defects. Congenital VMs are always present at birth, although they are not always visible. In contrast to vascular tumors, vascular malformations do not have a growth phase, nor an involution phase, they tend to grow proportionately with the child, never regressing and persisting throughout life [20]. Vascular malformations can be divided in slow-flow VM, fast-flow VM and complex-combined VM. Clinically, diagnosis of superficial VM is not difficult, deep VM should be determined by clinical examination with identification of postural movement and a positive puncture test. Ultrasound and MRI can aid significantly in the diagnosis of $\mathrm{VM}$, while angiography is usually reserved for treatment planning.

In our case, the patient had a slow growing tumor in the head and neck region over a period of more than two years. MRI findings were suggestive of a benign non-infiltrating tumor with heterogeneous signal intensity extending from the parotid to the parapharyngeal space which demonstrated intense enhancement after the administration of contrast material. Overall, the cystic nature of the tumor in conjunction with excess blood material in the fine needle aspirate led to the misdiagnosis of this GCA case as a vascular malformation. Sclerotherapy, as expected, had no effect on the lesion which in fact continued to slowly grow. Final diagnosis was only feasible after referral of the patient for surgical removal and subsequent histopathological and immunohistochemical testing of the tumor.

GCA is typically regarded as a non-invasive neoplasm with no potential for metastatic disease compared to solitary fibrous tumor which may rarely metastasize [19]. There is controversy regarding the potential of the tumor for recurrence. Since there have not been enough reports of GCA to allow us to come to any definitive

Table 2 Summary of World Health Organization (WHO) classification of soft tissue tumors of the neck

\begin{tabular}{|c|c|c|c|c|}
\hline Histologic type & Benign & $\begin{array}{l}\text { Intermediate, } \\
\text { locally aggressive }\end{array}$ & $\begin{array}{l}\text { Intermediate, } \\
\text { rarely metastasizing }\end{array}$ & Malignant \\
\hline Adipocytic & $\begin{array}{l}\text { Lipoma and its variants } \\
\text { (lipoblastoma, hibernoma, } \\
\text { lipomatosis) }\end{array}$ & $\begin{array}{l}\text { Atypical lipomatous } \\
\text { tumor, well-differentiated } \\
\text { liposarcoma }\end{array}$ & $\ldots$ & Liposarcoma \\
\hline $\begin{array}{l}\text { Fibroblastic/ } \\
\text { myofibroblastic }\end{array}$ & $\begin{array}{l}\text { Fibromatosis colli, myofibroma, } \\
\text { giant cell angiofibroma }\end{array}$ & Desmoid-type fibromatosis & $\begin{array}{l}\text { Solitary fibrous tumor } \\
\text { hemangio-pericytoma, } \\
\text { inflammatory myofibroblastic } \\
\text { tumor (inflammatory pseudotumor) }\end{array}$ & Fibrosarcoma \\
\hline $\begin{array}{l}\text { So-called } \\
\text { fibrohistiocytic }\end{array}$ & $\begin{array}{l}\text { Benign fibrous histiocytoma, } \\
\text { diffuse-type giant cell tumor } \\
\text { (pigmented villonodular } \\
\text { synovitis) }\end{array}$ & $\cdots$ & Giant cell tumor of soft tissues & $\begin{array}{l}\text { Malignant fibrous } \\
\text { histiocytoma (undifferentiated } \\
\text { pleomorphic sarcoma) }\end{array}$ \\
\hline Skeletal muscle & Rhabdomyoma & $\ldots$ & $\ldots$ & Rhabdomyosarcoma \\
\hline Smooth muscle & Leiomyoma, angioleiomyoma & $\ldots$ & $\ldots$ & Leiomyosarcoma \\
\hline Vascular & Hemangioma, lymphangioma & $\begin{array}{l}\text { Kaposiform } \\
\text { hemangioendothelioma }\end{array}$ & Kaposi sarcoma & Angiosarcoma \\
\hline Perivascular & Glomus tumor, myopericytoma & $\ldots$ & $\ldots$ & Malignant glomus tumor \\
\hline Chondro-osseous & Soft tissue chondroma & $\ldots$ & $\ldots$ & $\begin{array}{l}\text { Mesenchymal chondrosarcoma, } \\
\text { extraskeletal osteosarcoma }\end{array}$ \\
\hline $\begin{array}{l}\text { Uncertain } \\
\text { differentiation }\end{array}$ & Myxoma & $\ldots$ & Ossifying fibro-myxoid tumor & $\begin{array}{l}\text { Synovial sarcoma, alveolar } \\
\text { soft part sarcoma, } \\
\text { primitive neuroectodermal } \\
\text { tumor, Ewing sarcoma }\end{array}$ \\
\hline
\end{tabular}


conclusions regarding its long-term behavior, complete surgical removal and long-term follow up was considered the optimal treatment option.

Diagnosis of GCA is usually made after resection and immunohistochemical analysis. Histological characteristics of GCA include: (1) the presence of homogenous irregularly organized proliferating cells that are round or oval shaped (2) the stromal region that is often collagenized or myxoid and (3) the tumor is richly vascularized and contains pseudovascular spaces in the presence of interstitial hemorrhage. The pseudovascular spaces are lined with multinucleated giant cells [11].

The immunohistochemical features of GCA include positive staining for $\mathrm{CD} 34, \mathrm{CD} 99$, vimentin, variable bcl2 and negative staining for CD31, CD68, c-kit/CD117, muscle specific actin, S100, desmin $[7,21]$.

In our case, positive staining for CD34 in combination with the presence of giant cells and pseudovascular spaces confirmed the diagnosis of GCA. A controversial finding was the unexpected positivity of CD31 in the majority of cells compared to previous reports $[7,21]$. Clinically, this immunohistochemical finding may be interpreted by the more vascular nature of the tumor and it may provide a tool for differential diagnosis of GCA from SFT in the future. Nevertheless several other soft tissue tumors may present with similar findings. For example, GCA might be closely related to GCF, multinucleate cell angiohistiocytoma (MCA), SFT and VM.

GCF is usually differentially diagnosed from GCA by the fact that it is usually a poorly circumscribed lesion invading the dermis and subcutis of somatic soft tissues. The tumor, which typically occurs within the first decades of life, is rarely described in middle-aged and older patients. It most commonly affects the trunk [22]. Although GCF is composed of CD34 positive spindle and stellate shaped cells including multinucleated floretlike giant cells and tumor cell-lined pseudovascular spaces, it has infiltrative margins and has less conspicuous cellularity and vasculature. Clinically, GCF appears to be a more aggressive lesion than GCA in that up to $50 \%$ of cases locally recur [3].

MCA is a rare benign proliferation of the skin of unknown etiology. Clinically, MCA shows a predilection for adult women (F:M ratio 3:1) and manifests as a single or multiple firm, red-brown or violaceous papules with a smooth, or occasionally, scaly surface [23]. Histological examination shows a vascular proliferation predominantly of capillaries and veins in the upper and mid dermis with lymphohistiocyte infiltration. Multinucleate giant cells are arranged in a ring-like or overlapping pattern, with a positive staining for factor XIIIa [24].

The CD34-positive cell neoplasm most closely aligned to GCA is the SFT, which was first described in the pleura but has since been reported in many locations. In approximately half of all described cases it is located in the subcutaneous tissue, but also in deep soft tissue of the extremities, or in the head and neck region, the thoracic wall, mediastinum and abdominal cavity. SFTs on CT scans show moderate to intense enhancement in a well circumscribed mass. Histologically, SFTs show a patternless spindle cell proliferation in a collagenous stroma, although myxoid areas are not uncommon, and a hemangiopericytomatous vascular pattern that is not seen in GCA. Giant cells and pseudovascular spaces are infrequent and serve to distinguish this tumor type from GCA. Both are immunoreactive for CD34 and CD99 $[16,25,26]$.

There is a lot of debate in the literature concerning the classification of GCA. Although many authors believe GCA to be a distinct tumor with distinct histological and immunohistochemical characteristics, others support the opinion that this may be a variant of either SFT or GCA. In fact, the idea that GCA may be a variant of SFT is further supported by WHO's latest reclassification (2013) [27], where GCA is considered a synonym for extrapleural SFT rather than being a separate entity. In this reclassification GCA, SFT, hemangiopericytoma and lipomatous hemangiopericytoma are all interconnected and are included under the same category of 'extrapleural SFT'.

\section{Conclusion}

In conclusion, GCA is a rare benign tumor which mainly affects soft tissue(s) in the head and neck area. Clinical or radiological diagnosis alone is very difficult since clinical characteristics and radiological features of the tumor resemble those of many other benign soft tissue tumors. In particular, for cases resembling vascular malformations as in our report, clinicians should be aware of this entity to avoid unnecessary delay and treatment. Surgery is the main treatment. Microscopic examination and immunohistochemical analysis provide the only means of diagnosis mainly by identification of giant cells and pseudovascular spaces in conjunction with positivity for vimentin and CD34. Classification of GCA is still under question in our opinion. Further reports and studies of this tumor are needed to define the pathophysiology and understanding of the tumor.

\section{Consent}

Written informed consent was obtained from the patient for publication of this case report and any accompanying images. A copy of the written consent is available for review by the Editor-in-Chief of this journal.

\section{Abbreviations}

CKP: cytokeratin pan; CT: computed tomography; EMA: epithelial membrane antigen; GCA: giant cell angiofibroma; GCF: giant cell fibroblastoma; GFAP: glial fibrillary acidic protein; H\&E: hematoxylin and eosin;

MCA: multinucleate cell angiohistiocytoma; MRI: magnetic resonance imaging; MSA: muscle-specific actin; SFT: solitary fibrous tumor; SMA: smooth muscle actin; VM: vascular malformation; WHO: World Health Organization. 


\section{Competing interests}

The authors declare that they have no competing interests.

\section{Authors' contributions}

$\mathrm{YH}$ and EK wrote the paper. LW carried out the histological and immunohistochemical studies of the surgical specimens. GL and ZT were involved in collecting the clinical, imaging and laboratory data of the patient. $\mathrm{CZ}$ edited and modified the paper. All authors read and approved the final manuscript.

\section{Acknowledgements}

\section{Grant support}

This work was supported by generous grants from the National Natural Science Foundation of China (NSFC: 30973341, 81271112), Shu Guang Project (10SG19) supported by Shanghai Municipal Education Commission and Shanghai Education Development Foundation, the Development Foundation supported by Shanghai Municipal Human Resources and Social Security Bureau (201312), SMC Rising Star (A) Scholar supported by Shanghai Jiao Tong University.

\section{Author details}

'Department of Oral and Maxillofacial-Head and Neck Oncology, Ninth People's Hospital, Shanghai Jiao Tong University School of Medicine, 639, Zhi Zao Ju Road, Shanghai 200011, People's Republic of China. ${ }^{2}$ Department of Oral Pathology, Ninth People's Hospital, Shanghai Jiao Tong University School of Medicine, Shanghai, People's Republic of China.

Received: 10 December 2013 Accepted: 16 March 2014 Published: 24 April 2014

\section{References}

1. Dei Tos AP, Seregard S, Calonje E, Chan JK, Fletcher CD: Giant cell angiofibroma. A distinctive orbital tumor in adults. Am J Surg Pathol 1995, 19(11):1286-1293.

2. Rousseau A, Perez-Ordonez B, Jordan RC: Giant cell angiofibroma of the oral cavity: report of a new location for a rare tumor. Oral Surg Oral Med Oral Pathol Oral Radiol Endod 1999, 88(5):581-585.

3. Sigel JE, Fisher C, Vogt D, Goldblum JR: Giant cell angiofibroma of the inguinal region. Ann Diagn Pathol 2000, 4(4):240-244.

4. Yazici B, Setzen G, Meyer DR, Williams EF, McKenna BJ: Giant cell angiofibroma of the nasolacrimal duct. Ophthal Plast Reconstr Surg 2001, 17(3):202-206.

5. de Andrade CR, Lopes MA, de Almeida OP, Leon JE, Mistro F, Kignel S: Giant cell angiofibroma of the oral cavity: a case report and review of the literature. Medicina oral, patologia oral y cirugia bucal 2008, 13(9):E540-E543.

6. Gonzalez-Perez LM, Sanchez-Gallego F, Haro-Luna JJ, Infante-Cossio P: Giant cell angiofibroma of parapharyngeal space: a report of a new location for a rare tumour. Int J Oral Maxillofac Surg 2010, 39(10):1024-1027.

7. Shu HR, Yang QT, Lai YY, Mo JG, Mao WH, Song JX, Zheng GJ: Giant cell angiofibroma in the vocal cord. Chin Med J 2010, 123(23):3479-3481.

8. Pala EE, Beyhan R, Bayol U, Cumurcu S, Kucuk U: Giant cell angiofibroma in unusual localization: a case report. Case Rep Pathology 2012, 2012:408575.

9. Silverman JS, Tamsen A: A cutaneous case of giant cell angiofibroma occurring with dermatofibrosarcoma protuberans and showing bimodal CD34+ fibroblastic and FXIIla + histiocytic immunophenotype. J Cutan Pathol 1998, 25(5):265-270.

10. Husek K, Vesely K: Extraorbital giant cell angiofibroma. Cesk Patol 2002, 38(3):117-120.

11. Thomas R, Banerjee SS, Eyden BP, Shanks JH, Bisset DL, Hunt R, Byers RJ, Oogarah P, Harris M: A study of four cases of extra-orbital giant cell angiofibroma with documentation of some unusual features. Histopathology 2001, 39(4):390-396.

12. Guillou L, Gebhard S, Coindre JM: Orbital and extraorbital giant cell angiofibroma: a giant cell-rich variant of solitary fibrous tumor? Clinicopathologic and immunohistochemical analysis of a series in favor of a unifying concept. Am J Surg Pathol 2000, 24(7):971-979.

13. Fukunaga M, Ushigome S: Giant cell angiofibroma of the mediastinum. Histopathology 1998, 32(2):187-189.
14. Qian YW, Malliah R, Lee HJ, Das K, Mirani N, Hameed M: A t(12;17) in an extraorbital giant cell angiofibroma. Cancer Genet Cytogenet 2006, 165(2):157-160.

15. Kintarak S, Natiella J, Aguirre A, Brooks J: Giant cell angiofibroma of the buccal mucosa. Oral Surg Oral Med Oral Pathol Oral Radiol Endod 1999, 88(6):707-713.

16. Piperi E, Rohrer MD, Pambuccian SE, Koutlas IG: Vascular solitary fibrous tumor with 'floret' cells or giant cell angiofibroma? A lingual example highlighting the overlapping characteristics of these entities and positive immunoreaction for estrogen and progesterone receptors. Oral Surg Oral Med Oral Pathol Oral Radiol Endod 2009, 107(5):685-690

17. Mikami Y, Shimizu M, Hirokawa M, Manabe T: Extraorbital giant cell angiofibromas. Mod Pathol 1997, 10(11):1082-1087.

18. Sonobe H, Iwata J, Komatsu T, Fukushima A, Hayashi N, Moriki T, Shimizu K, Ohtsuki Y: A giant cell angiofibroma involving 6q. Cancer Genet Cytogenet 2000, 116(1):47-49.

19. Razek AA, Huang BY: Soft tissue tumors of the head and neck: imaging-based review of the WHO classification. Radiographics 2011, 31(7):1923-1954. doi:10.1148/rg.317115095.

20. Glowacki J, Mulliken JB: Mast cells in hemangiomas and vascular malformations. Pediatrics 1982, 70(1):48-51.

21. Farmer JP, Lamba M, McDonald H, Commons AS: Orbital giant cell angiofibroma: immuno-histochemistry and differential diagnosis. Can J Ophthalmol 2006, 41(2):216-220.

22. Fletcher CD: Giant cell fibroblastoma of soft tissue: a clinicopathological and immunohistochemical study. Histopathology 1988, 13:499-508.

23. Belgodere X, Wechsler J, Pasqualini G, Paoli M: Multinucleate cell angiohistiocytoma. Ann Dermatol Venereol 1999, 126(5):431-432.

24. Jaconelli L, Kanitakis J, Ktiouet S, Faure M, Claudy A: Multinucleate cell angiohistiocytoma: report of three new cases and literature review. Dermatol Online J 2009, 15(2):4.

25. Krishnakumar S, Subramanian N, Mohan ER, Mahesh L, Biswas J, Rao NA: Solitary fibrous tumor of the orbit: a clinicopathologic study of six cases with review of the literature. Surv Ophthalmol 2003, 48:544-554.

26. Weiss SW, Goldblum JR: Soft tissue tumors of intermediate malignancy of uncertain type. In Enzinger and Weiss's Soft Tissue Tumors. Edited by Weiss SW, Goldblum JR. St. Louis: Elsevier Mosby; 2008:1093-1160.

27. Soft tissue tumours. In World Health Organization Classification of Tumours. Pathology and Genetics of Tumours of Soft Tissue and Bone. Edited by Fletcher CDM, Unni KK, Mertens F. Lyon: IARC Press; 2002.

doi:10.1186/1477-7819-12-117

Cite this article as: He et al:: Giant cell angiofibroma misdiagnosed as a vascular malformation and treated with absolute alcohol for one year: a case report and review of the literature. World Journal of Surgical Oncology 2014 12:117.

\section{Submit your next manuscript to BioMed Central and take full advantage of:}

- Convenient online submission

- Thorough peer review

- No space constraints or color figure charges

- Immediate publication on acceptance

- Inclusion in PubMed, CAS, Scopus and Google Scholar

- Research which is freely available for redistribution 